\title{
Beyond the lab
}

\section{Virtual reality for undergraduate Anatomy and Physiology students}

\section{C}

urrent teaching practice in undergraduate higher education anatomy and physiology courses incorporates the use of various instructional methodologies to reinforce the anatomical relationships between structures. ${ }^{1,2}$ These methods can include basic handson physical models, human and animal dissection labs, and interactive technology. Technological advances continue to drive the production of innovative anatomy and physiology electronic tools, including:

- virtual dissection in 3-D (e.g., Virtual Dissection Boards from Anatomage, 3D4Medical, and Anatomy.TV),

- augmented reality (AR) (e.g., Human Anatomy Atlas),

- mixed reality (e.g., Microsoft HoloLens Case Western Reserve Medical School and Cleveland Clinic digital anatomy app), and

- 3-D virtual reality (VR) (e.g., 3D Organon VR Anatomy and YOU by Sharecare apps).

In general, the use of technology in anatomy and physiology courses allows students to view structures and physiological concepts in an electronic environment without the use of a cadaver. Virtual dissection in 3-D does exactly what the name describes: students virtually dissect the body using 3-D technology on devices (tablets, computers, etc.) and specially designed tables. AR differs from 3-D virtual dissection in that it superimposes static digital information on realworld images, which usually occurs through tablets or smartphones. Whereas mixed reality allows the user to see their physical environment while manipulating interactive virtual 3-D objects. Virtual reality allows the user to experience a computer-generated immersive and interactive 3-D environment with the use of a headset, laptop with apps, and controllers. ${ }^{3}$ Several studies suggest that VR is superior to other 3-D and AR forms as it provides a 360-degree spatial knowledge experience similar to real life and enhances students' motivation for learning the content. ${ }^{4,5}$

Academic libraries continue to be on the forefront of technological adoption in support of curricular changes and provide an ideal environment for out-of-classroom content engagement. There are an increasing number of libraries expanding their services to include circulating VR equipment. This offers greater access for multiple departments that otherwise lack funding for innovative technology. ${ }^{6,7}$ Moreover, academic health sciences libraries have started to collect VR apps and equipment for use in medical education. VR apps include 3D Organon VR Anatomy, YOU by Sharecare, Osso VR (surgical practice), and EmbodiedLabs (empathy training). ${ }^{89}$

In 2014, the Penn State-Hazleton head librarian and a biology professor formed a partnership to purchase iPads with anatomy apps for student use in the laboratory and library. ${ }^{10}$ Building on the success of this project, librarians from the Penn State University main campus invited the Hazleton research team to participate in a study that would use VR technology for anatomy and physiology undergraduate education. In 2018, the research team received a Health Sciences Library Project Award from the National Network of Libraries of Medicine Mid-Atlantic Region to purchase VR equipment and software for use in the laboratory and library.

Kathleen Phillips is Nursing and Allied Health liaison librarian at Penn State University, email: kec5013@ psu.edu, Valerie A. Lynn is head librarian at Penn State-Hazleton, email: vag3@psu.edu, Amie Yenser is assistant teaching professor of Biology at Penn StateHazleton, email: alv10@psu.edu, Christina Wissinger is Health Sciences librarian at Penn State University, email: clw68@psu.edu

(C) 2021 Kathleen Phillips, Valerie A. Lynn, Amie Yenser, and Christina Wissinger 
The objectives of this study were threefold: 1) to provide an immersive, virtual human dissection experience for anatomy and physiology students, 2) to explore the possibility of VR improving student exam scores in the anatomy and physiology courses, and 3) to provide continued support of VR technology.
- six Alienware Laptop Computers and carrying cases ( 2 in the biology lab and 4 in the library for 24hour checkout),

- two large-screen monitors with wall mounts (2 in library group study rooms as the biology lab already contained large screen monitors),

- six sets of VR hardware (Acer Windows Mixed
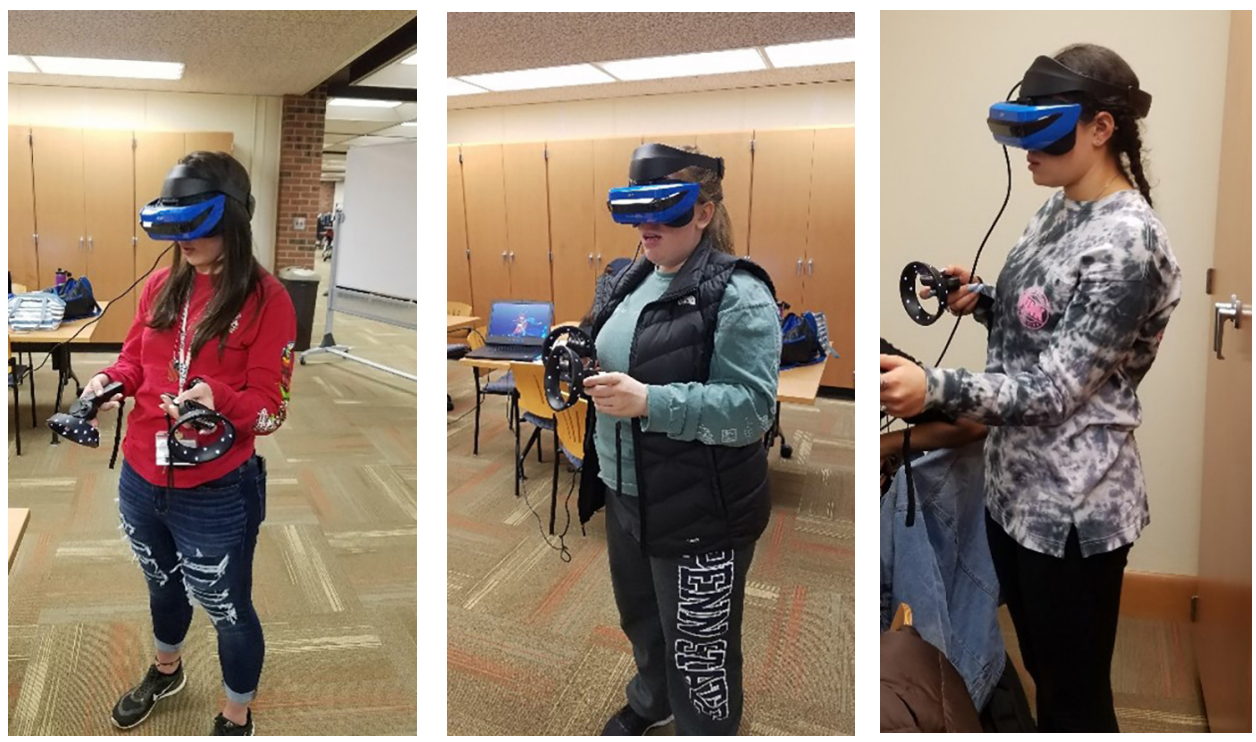

Students using the Acer Windows Mixed Reality Headset with motion controllers.

\section{Identifying the appropriate VR tech}

Several different types of VR tools were tested: HTC Vive, Microsoft HoloLens, and Acer Mixed Reality Headset. Two of these technologies had limitations that made them unsuitable for this project. HTC Vive required a ceiling-mounted sensor that restricted the use of this technology to a single space. The Microsoft HoloLens headset was uncomfortable and projected objects at odd heights. Acer Mixed Reality Headset proved to be most useful for this study. It was portable, comfortable, affordable, and projected all VR images in the immediate vicinity. The authors tested two educational VR software apps that met the curriculum criteria for the anatomy and physiology courses: 3D Organon VR Anatomy app and the Case Western Reserve University Medical School and the Cleveland Clinic software. The 3D Organon VR Anatomy app was selected for this study as it contained all organ systems, included dissection, and projected images within a comfortable visual range. The following equipment was purchased and installed for student and biology professor use:
Reality Headset with motion controllers) and 6 carrying cases ( 2 in the biology lab and 4 in the library for 24-hour checkout), and

- six copies of the 3D Organon VR Anatomy software (installed on each laptop).

\section{Using VR in the classroom and library}

VR technology was introduced to Penn State-Hazleton students enrolled in the fall 2018 anatomy course (BIOL 129, n=51) and spring 2019 physiology course (BIOL 141/142, n=71). Both courses were taught by the same biology professor. Students learned how to use the VR equipment during the biology laboratory sessions and then worked in the library to complete group assignments.

During the fall 2018 semester, anatomy students used the VR Organon app to study bones, musculature, and the nervous system during laboratory course sessions. The use of this app was required to complete a graded group assignment in which students virtually dissected the brain while following a checklist of struc- 
tures. Students within each group determined who would wear the headset (one individual or shared responsibility), while the others watched the dissection on a large-screen monitor in a library study room. Students completed this assignment without the oversight of the biology professor.

At times, students experienced technical difficulties using the VRequipment and software. Equipment setup required approximately 15 minutes to physically connect cables, connect to wi-fi, and run the respective VR app. Students, the biology professor, and librarians frequently performed minor troubleshooting activities (connecting to apps, pairing the controllers, etc.). Students found the Organon app difficult to navigate. At times they were virtually trapped inside the skeleton, unable to move outside the ribcage. The solution involved a ten-minute process of resetting the headset boundaries thus limiting actual learning time.

Based on fall 2018 student experiences, the biology professor researched additional VR anatomy/physiology apps and discovered the free YOU by Sharecare app. The app was easier to navigate and offered more clinical aspects for exploration.

The new app was used in the biology laboratory by students in the 2019 physiology course. During completion of an extra-credit graded group assignment, students were required to write short paragraphs that described their VR experience using the YOU app to introduce and treat clinical disease manifestations. For example, students created a coronary blockage and treated it with a stent. They also viewed the difference in bronchiole diameter after asthma was treated with a virtual inhaler. The extra credit assignment took place in the library and the VR equipment was pre-prepared for use to offset technical difficulties. The biology professor was present in the library to assist with potential technical difficulties as students completed the assignment. Anatomy students who experienced frustration with the VR equipment in the fall semester were apprehensive about using it in the spring physiology course. However, when these students used the YOU app, they were more engaged and excited about exploring organ functionality.

\section{Student experiences}

To quantify the student VR experience, an anonymous survey (Likert scale with sections for comments) was distributed to each student at the end of the fall semester during lecture. At the end of the spring semester another student survey was distributed to determine student VR experiences.
Spring semester survey questions were similar to fall semester survey questions. The only difference between the two surveys were questions about the YOU app instead of the Organon app.

On average, students ranked the YOU app higher than the Organon app. Most notably, students found YOU to be more beneficial for virtual dissection, ranking it 2.02 points higher than Organon. As an educational tool, YOU was ranked higher in the "I did better on exams $\mathrm{b} / \mathrm{c}$ of the VR technology" (+2.03), "It was beneficial to use Virtual dissection" (+2.02), and "The VRassignment helped me learn the material" $(+1.71)$ questions. YOU received a lower score, not for perceived educational advantages, but for ease of access from the library (-1.04). This lower score could be attributed to the pre-prepared VR stations as they might have contributed to student perception of limited VR equipment accessibility in the library.

Average lab 3 and lecture 3 exam scores over a threeyear period were assessed, as well. Exam 3 assessed the systems and concepts students explored within the VR apps. Biology 129 students' exam scores improved slightly from 2017-2018 (+1.8\%, Lab Exam and +2.53\% Lecture Exam), but scores did not improve for Biology 141/142 (-11.2\% Lab Exam; -1.18\% Lecture Exam).

It is unclear if the Biology 129 students exam scores' improvement (from the fall 2017 to fall 2018 semesters) or if the decline in Biology 141/142 student's scores (from the spring 2018 to spring 2019 semesters) were influenced by the addition of VR as an educational component of the courses. Student populations fluctuate (incoming GPAs, maturity/motivation, etc.) making it difficult to draw conclusions about student performance from only one year of data. Future research could include a longitudinal study to determine the statistically significant versus perceived impact of VR on overall course performance.

A supplemental document, including a table of student survey results and figures representing Biology 129 and Biology 141/142 average scores, can be found in our institutional repository. ${ }^{11}$

VR provided an engaging and realistic view of the human body beyond what was previously available in Penn State-Hazleton anatomy and physiology courses. Based on the survey data, students during the fall semester reported their VR experience with Organon as less than ideal. However, spring students felt that both their learning experience and exam performance were enhanced by theYOU app. As reported in qualitative survey responses, students particularly liked the ability to introduce and treat 
disorders while virtually standing in the affected organ. It provided a new perspective and integrated concepts taught in the laboratory and lecture. Similar to other studies, some Penn State-Hazleton students suffered from motion sickness while using VR ${ }^{12,13}$ This is an important factor to consider when incorporating VR into a classroom environment. Overall, VR technology increased student engagement and motivated them to explore immersive 3-D physiological functioning.

\section{Conclusion}

Demonstrated by this project, VR provides an engaging realistic view of the human body beyond what is typically available in undergraduate anatomy and physiology courses. The Penn State-Hazleton students especially liked the ability to introduce and treat disorders while virtually standing in the affected organ in the YOU app. It gave them a new perspective and integrated concepts taught in the laboratory and lecture. Even though exam scores were not substantially affected by the use of VR, positive student experiences presented a compelling case for its continued use in the anatomy and physiology curriculum.

\section{Acknowledgements}

Developed resources reported in this [publication, press release, Internet site] are supported by the National Library of Medicine (NLM), National Institutes of Health (NIH) under cooperative agreement number UG4LM012342 with the University of Pittsburgh, Health Sciences Library System. The content is solely the responsibility of the authors and does not necessarily represent the official views of the National Institutes of Health.

\section{Notes}

1. Mohamed Estai and Stuart Bunt, "Best teaching practices in anatomy education: A critical review," Annals of Anatomy 208, November (2016):151-157, https://doi.org/10.1016/j.aanat.2016.02.010.

2. Chien-Huan Chien, Chien-Hsu Chen, TaySheng Jeng, "An interactive augmented reality system for learning anatomy structure," Proceedings of the International MultiConference of Engineers and Computer Scientists, Hong-Kong, China March 17-19, 2010.

3. Tara J. Brigham, "Reality check: Basics of augmented, virtual, and mixed reality," Medical Reference
Services Quarterly36, no. 2 (2017):171-178, https:// doi.org/10.1080/02763869.2017.1293987.

4. Christian Moro, Zane Štromberga, Achanasios Raikos, Allan Stirling, "The effectiveness of virtual and augmented reality in health sciences and medical anatomy," Anatomical Sciences Education,10, no. 6 (2017):549-559, https://doi.org/10.1002/ase.1696.

5. Christian Moro, Sue Gregory, "Utilising anatomical and physiological visualisations to enhance the face-to-face student learning experience in biomedical sciences and medicine (The Book) in Biomedical visualization," (Switzerland: Springer Nature, 2019):4148, https://doi.org/10.1007/978-3-030-19385-0_3.

6. Anne Llewellyn, "Innovations in learning and teaching in academic libraries: A literature review," New Review of Academic Librarianship 25, no. 2-4 (2020):129-149, https://doi.org/10.1080/13614533 .2019 .1678494 .

7. Brandon Patterson, Tallie Casucci, Bryan Elias Hull, Nancy T. Lombardo, "Library as the technology hub for the health sciences," Medical Reference Services Quarterly37, no. 4 (2018):341-356, https://doi.org/ 10.1080/02763869.2018.1514899.

8. Jason Lilly, Kellie N, Kaneshiro, Chelsea Misquith, Brandon Dennett, "Creating a new 'reality' for medical education: The nexus reality lab for virtual reality," Journal of the Medical Library Association 107, no. 4 (2019):609-610.

9. Elizabeth Dyer, Barbara J. Swartzlander, Marilyn R. Gugliucci, "Using virtual reality in medical education to teach empathy," Journal of the Medical Library Association 106, no. 4 (2018):498-500.

10. Valerie A. Lynn, Amie Yenser, Ron Harman, "Body apps: iPads for undergrad Anatomy and Physiology students," C\&RL News 76, no 3 (2015):149-152.

11. Kathleen Phillips, Valerie A. Lynn, Amie Yenser, Christina L. Wissinger, "Virtual reality for undergraduate Anatomy and Physiology students: Supplemental Table and Figures," (2020): prepub, https://doi.org/10.26207/2sd2-7239.

12. GyuChang Lee, "Full-immersion virtual reality: Adverse effects related to static balance," Neuroscience Letters April (2020): prepub, https://doi. org/10.1016/j.neulet.2020.134974.

13. John F. Golding, "Motion sickness susceptibility," Autonomic Neuroscience 129, no. 1-2 (2006):67-76, https://doi.org/10.1016/j. autneu.2006.07.019. 22 